\title{
Recent changes in the American Joint Commission Cancer staging of human papilloma virus related oropharyngeal cancer: validating the present and envisioning the future
}

\author{
Brett A. Miles \\ Department of Otolaryngology Head and Neck Surgery, Icahn School of Medicine at Mount Sinai, New York, NY, USA \\ Correspondence to: Brett A. Miles, DDS, MD. Department of Otolaryngology Head and Neck Surgery, Icahn School of Medicine at Mount Sinai, \\ New York, NY, USA. Email: Brett.miles@mountsinai.org. \\ Comment on: Geltzeiler M, Bertolet M, Albergotti W, et al. Staging HPV-related oropharyngeal cancer: Validation of AJCC-8 in a surgical cohort. \\ Oral Oncol 2018;84:82-7.
}

Submitted Dec 06, 2018. Accepted for publication Dec 11, 2018.

doi: $10.21037 /$ cco.2018.12.01

View this article at: http://dx.doi.org/10.21037/cco.2018.12.01

The dramatically increasing incidence of human papilloma virus (HPV) related oropharyngeal squamous cell carcinoma (OPSCC) continues to capture the scientific and clinical interest of the head and neck oncologic world. While the oncologic potential of HPV has been recognized for decades in a variety of anatomical subsites, most notably the cervix, the dramatic rise in the incidence of oropharyngeal cancers related to HPV is a somewhat more recent development. In fact, recent data indicates that the numbers of HPV related head and neck cancer are increasing and has surpassed other anatomic sites in terms of incidence (1). Of interest is the fact that we currently have a vaccine which, if adequate penetration of the population was achieved, could prevent $90 \%$ of these malignancies (1). Nevertheless, at the present time we are in the unique position of having recently recognized a dramatically increasing prevalence of a cancer with a well characterized oncogenic pathway and pathogen, as well as a highly effective preventative vaccine, with a lack of real ability to decrease the incidence of the disease in the near term. This is primarily due to a largely unvaccinated population potentially exposed to oncogenic strains of HPV, coupled with a vaccination strategy in its infancy. Therefore, the treatment of HPV related oropharyngeal cancer will continue to dominate the head and neck oncologic community for the foreseeable future.

Perhaps the most important discovery regarding the treatment of HPV related OPSCC population, was the fact that with traditional therapy, the prognosis was markedly improved related to oropharyngeal cancers with environmental etiology such as tobacco and alcohol, which has been validated in recent meta-analysis $(2,3)$. This discovery lead to the recognition that the American Joint Commission Cancer (AJCC) $7^{\text {th }}$ edition staging system, which was based on data in the "pre-HPV" era, was inadequate to appropriately stratify patients with HPV related OPSCC, primarily due the lack of survival impact with advanced cervical nodal burden in this population (4). This discovery lead to the development and implementation of the current AJCC $8^{\text {th }}$ edition staging system in 2018 , which for the first time, staged HPV related OPSCC as a distinct disease, and separate from environmentally related HPV negative OPSCC (5). This new staging system was developed from both surgical and non-surgical cohorts, in order to elucidate both clinical and pathological factors in this population which lead to the development of a staging system with improved survival discrimination (6-8). The impact of HPV status, and other clinical and pathologic factors resulted in the simplification of the TNM system, and resulted in the elimination of stage IV locoregional disease, with stage IV being reserved the presence of distant metastasis. It has become widely accepted that the new AJCC $8^{\text {th }}$ edition, while imperfect, was a major leap forward in terms of the stratification of survival for OPSCC (8-12).

Several investigators have published recent validation studies from a variety of data from different centers in order to validate the $8^{\text {th }}$ edition staging system, and ensure that the appropriate survival stratification observed in the original data used to create the staging system was 
maintained (12-17). Recently, Geltzeiler et al., examined a surgical cohort of 309 patients at two institutions (University of Pittsburgh, and Oregon Health Sciences University) as part of this validation effort. Patients treated with upfront transoral surgery (robotic, laser, and traditional) were included in the analysis and the performance of the AJCC $7^{\text {th }}$ and AJCC $8^{\text {th }}$ editions in terms of overall survival (OS) and disease-free survival (DFS) were compared (11). Multivariate Cox proportional hazard regression models with AIC bootstrapping methods were used to create comparisons of the performance of the staging systems. As has been shown in other recent reports, the authors found that in this cohort, the AJCC $7^{\text {th }}$ edition staging system for OPSCC did not adequately discriminate OS or DFS appropriately and was outperformed by the AJCC $8^{\text {th }}$ edition staging system. The clinical and pathological staging characteristics were also non-discriminatory when the AJCC $7^{\text {th }}$ edition staging system was applied, in contrast to the AJCC $8^{\text {th }}$ edition. In terms of the comparison of the improved performance of the AJCC $8^{\text {th }}$ edition system, the authors utilized the Akaike's Information Criteria (AIC) which allowed the staging models to be compared, and the AJCC $8^{\text {th }}$ edition model did exhibit a better fit for predicting OS and DFS. This investigation, which was one of the largest surgical cohorts used in validation, and those cohorts noted previously appear to confirm the improved performance of the AJCC $8^{\text {th }}$ edition staging system for HPV related OPSCC. The authors also correctly note that the purpose of a staging system is to stratify cohorts in terms of prognosis, not to drive therapeutic decisions. This is a critical message to impart when evaluating the staging literature; that changes in treatment paradigms should be driven by the results of clinical trials examining the impact of treatment strategies not by the staging system alone. The AJCC $8^{\text {th }}$ edition staging, with its improved stratification of survival outcomes, will doubtless drive current and future clinical trials designed improve outcomes for patients with HPV related OPSCC. The work of Geltzeiler et al. as well as similar high-quality validation studies, is the key to unlocking the potential of the new AJCC $8^{\text {th }}$ edition staging system, and this work is critical when implementing changes in staging systems with worldwide implications.

One of the unique aspects of the AJCC $8^{\text {th }}$ edition staging system is the separation of clinical and pathologic staging, which in theory should provide increased discrimination of survival outcomes. Interestingly, Geltzeiler et al. found that both clinical and pathological $8^{\text {th }}$ edition staging performed similarly in terms of OS but noted that pathological staging outperformed the clinical staging in terms of DFS. This has implications in future surgical trial design, in terms of treatment arm assignment. In addition, it highlights one of the weaknesses of many of the validation studies performed in surgical cohorts, primarily surgical selection/ treatment selection bias $(18,19)$. In addition, while this is one of the largest cohorts in the literature, the inclusion of traditional surgery in an era prior to the recognition of $\mathrm{HPV}$ as an oncologic driver in OPSCC (the study included patients from 1983-2015) further clouds the issue of selection bias in surgical cohorts. Additional validation of the AJCC $8^{\text {th }}$ edition clinical staging system in large cohorts of patients treated in a similar fashion as the patients in the International Collaboration on Oropharyngeal cancer Network for Staging (ICON-S) cohort (i.e., non-surgical) used to create the staging is needed $(16,18)$. Retrospective cohort comparison is somewhat limited in terms of statistical power when logistic regression analysis technique is utilized, if low subject numbers exist in comparative groups. This fact provides some insight into the confidence intervals reported in the study. In addition, this investigation and others while supporting the validity of the new staging system have resulted in several additional questions which remain related to the staging and treatment of HPV related OPSCC. Perhaps most notably absent in the $8^{\text {th }}$ edition staging system is the inclusion of smoking in the setting of HPV positive disease, a known negative risk factor (20). Questions related to pathologic staging criteria remain including the presence of "close margins", the impact of the presence and magnitude of extra nodal extension (in both surgical and non-surgical cohorts), and the magnitude of the impact of traditional pathologic criteria such as perineural invasion, and lymphovascular invasion $(9,21)$. These questions warrant additional investigation regarding the evaluation of clinical and pathologic factors in the HPV OPSCC era which might impact future staging systems.

Perhaps the most notably absent criteria in the AJCC $8^{\text {th }}$ edition staging system is the molecular characterization of HPV related OPSCC for staging stratification. While this is not a condemnation of the AJCC $8^{\text {th }}$ edition staging system, it is an observation related to the paucity of high-quality molecular data associated with this disease. We live in an age of molecular diagnostics, and certainly many molecular techniques are currently being applied in a robust fashion to determine prognosis, identify novel therapies, and stratify patients for clinical trials in a variety of solid tumors. While the current AJCC $8^{\text {th }}$ staging system represents a dramatic leap forward for head and neck oncologists who treat patients 
with HPV related head and neck cancer, we lag behind other solid tumors in the molecular characterization of these patients. In fact, the TNM system and even the updated AJCC $8^{\text {th }}$ staging is somewhat crude when viewed from a molecular perspective. Recent work evaluating a variety of molecular markers such as programmed cell death 1 (PD-1)/ programmed death ligand 1 (PD-L1), and tumor molecular and immune profiling provide a promising approach for future prognostic prediction and therapy (22-24). Future efforts should focus on tumor biorepositories with linked data as well as further molecular and immune characterization of HPV related tumors in order to facilitate stratification of HPV related OPSCC patients, rather than merely separating OPSCC in a binary fashion based on HPV status. There is little doubt that HPV related OPSCC represents a heterogenic disease and is currently poorly characterized from a molecular prognostic and therapeutic standpoint. Improved understanding of the molecular drivers, the tumor immune microenvironment, and the unique aspects of HPV infection in the oropharynx will doubtless lead to further improvements in future staging systems, and available therapy for the coming wave of patients afflicted with this disease.

\section{Acknowledgments}

None.

\section{Footnote}

Conflicts of Interest: The author has no conflicts of interest to declare.

\section{References}

1. Razzaghi H, Saraiya M, Thompson TD, et al. Five-year relative survival for human papillomavirus-associated cancer sites. Cancer 2018;124:203-11.

2. Ang KK, Harris J, Wheeler R, et al. Human papillomavirus and survival of patients with oropharyngeal cancer. N Engl J Med 2010;363:24-35.

3. Sun G, Dong X, Tang X, et al. The prognostic value of HPV combined p16 status in patients with anal squamous cell carcinoma: a meta-analysis. Oncotarget 2017;9:8081-8.

4. Klozar J, Koslabova E, Kratochvil V, et al. Nodal status is not a prognostic factor in patients with HPV-positive oral/ oropharyngeal tumors. J Surg Oncol 2013;107:625-33.

5. Amin MB, Greene FL, Edge SB, et al. The Eighth
Edition AJCC Cancer Staging Manual: Continuing to build a bridge from a population-based to a more "personalized" approach to cancer staging. CA Cancer J Clin 2017;67:93-9.

6. Haughey BH, Sinha P, Kallogjeri D, et al. Pathologybased staging for HPV-positive squamous carcinoma of the oropharynx. Oral Oncol 2016;62:11-9.

7. O'Sullivan B, Huang SH, Su J, et al. Development and validation of a staging system for HPV-related oropharyngeal cancer by the International Collaboration on Oropharyngeal cancer Network for Staging (ICON-S): a multicentre cohort study. Lancet Oncol 2016;17:440-51.

8. Lydiatt WM, Patel SG, O'Sullivan B, et al. Head and Neck cancers-major changes in the American Joint Committee on cancer eighth edition cancer staging manual. CA Cancer J Clin 2017;67:122-37.

9. Sharma SJ, Wagner S, Reder HS, et al. The 8th edition AJCC/UICC TNM staging for p16-positive oropharyngeal carcinoma: is there space for improvement? Eur Arch Otorhinolaryngol 2018;275:3087-91.

10. Hobelmann KC, Topf MC, Bar-Ad V, et al. AJCC-8ed nodal staging does not predict outcomes in surgically managed HPV-associated oropharyngeal cancer. Oral Oncol 2018;82:138-43.

11. Geltzeiler M, Bertolet M, Albergotti $W$, et al. Staging HPV-related oropharyngeal cancer: Validation of AJCC-8 in a surgical cohort. Oral Oncol 2018;84:82-7.

12. Zhan KY, Eskander A, Kang SY, et al. Appraisal of the AJCC 8th edition pathologic staging modifications for HPV-positive oropharyngeal cancer, a study of the National Cancer Data Base. Oral Oncol 2017;73:152-9.

13. Horne ZD, Glaser SM, Vargo JA, et al. Confirmation of proposed human papillomavirus risk-adapted staging according to AJCC/UICC TNM criteria for positive oropharyngeal carcinomas. Cancer 2016;122:2021-30.

14. Husain ZA, Chen T, Corso CD, et al. A Comparison of Prognostic Ability of Staging Systems for Human Papillomavirus-Related Oropharyngeal Squamous Cell Carcinoma. JAMA Oncol 2017;3:358-65.

15. Malm IJ, Fan CJ, Yin LX, et al. Evaluation of proposed staging systems for human papillomavirus-related oropharyngeal squamous cell carcinoma. Cancer 2017;123:1768-77.

16. Mizumachi T, Homma A, Sakashita T, et al. Confirmation of the eighth edition of the AJCC/UICC TNM staging system for HPV-mediated oropharyngeal cancer in Japan. Int J Clin Oncol 2017;22:682-9.

17. Sano D, Yabuki K, Arai Y, et al. The applicability of new 
TNM classification for humanpapilloma virus-related oropharyngeal cancer in the 8th edition of the AJCC/ UICC TNM staging system in Japan: A single-centre study. Auris Nasus Larynx 2018;45:558-65.

18. Huang SH, Hahn E, Tsang RK, et al. The interplay of IMRT and transoral surgery in HPV-mediated oropharyngeal cancer: Getting the balance right. Oral Oncol 2018;86:171-80.

19. Badhey AK, Olson A, Kadakia S, et al. Application of the Eighth Edition American Joint Committee on Cancer Staging System for HPV-Related Oropharyngeal Cancer Treated With Transoral Robotic Surgery. Laryngoscope 2018;128:1133-9.

20. Haigentz M Jr, Suarez C, Strojan P, et al. Understanding Interactions of Smoking on Prognosis of HPV-Associated Oropharyngeal Cancers. Adv Ther 2018;35:255-60.

21. Persky MJ, Albergotti WG, Rath TJ, et al. Positive

Cite this article as: Miles BA. Recent changes in the American Joint Commission Cancer staging of human papilloma virus related oropharyngeal cancer: validating the present and envisioning the future. Chin Clin Oncol 2019;8(Suppl 1):S6. doi: $10.21037 /$ cco.2018.12.01
Margins by Oropharyngeal Subsite in Transoral Robotic Surgery for T1/T2 Squamous Cell Carcinoma. Otolaryngol Head Neck Surg 2018;158:660-6.

22. Stepp WH, Farquhar D, Sheth S, et al. RNA Oncoimmune Phenotyping of HPV-Positive p16Positive Oropharyngeal Squamous Cell Carcinomas by Nodal Status. JAMA Otolaryngol Head Neck Surg 2018;144:967-75.

23. Yang WF, Wong MC, Thomson PJ, et al. The prognostic role of PD-L1 expression for survival in head and neck squamous cell carcinoma: A systematic review and metaanalysis. Oral Oncol 2018;86:81-90.

24. Otter S, Whitaker S, Chatterjee J, et al. The Human Papillomavirus as a Common Pathogen in Oropharyngeal, Anal and Cervical Cancers. Clin Oncol (R Coll Radiol) 2019;31:81-90. 